\title{
APPENDIX C: EXPERTISE OF AUTHORS
}

\section{Bogdan Constantinescu}

Horia Hulubei National Institute for Nuclear Physics and Engineering, Bucharest, Romania. XRF and micro-PIXE analysis of archaeological artefacts and geological samples.

\section{Bernhard Blümich}

Professor of Macromolecular Chemistry at the RWTH Aachen University, Germany. Specialist in nuclear magnetic resonance instrumentation, methods, and applications to materials and processes.

\section{Dimiter L. Balabanski}

Extreme Light Infrastructure - Nuclear Physics, Horia Hulubei National Institute for R\&D in Physics and Nuclear Engineering, Bucharest-Magurele, Romania. Senior researcher, Head of research on nuclear physics and applications with high-brilliance gamma-beams; expertise in experimental nuclear physics and applications.

\section{Alicja Chruścińska}

Institute of Physics, Faculty of Physics, Astronomy and Informatics, Nicolaus Copernicus University, Toruń, Poland. Specialist in luminescent studies and dating of archaeological artefacts.

\section{Mariaelena Fedi}

National Institute of Nuclear Physics (INFN), Florence, Italy. Physicist/technologist working in applied nuclear physics. Expertise in radiocarbon dating and IBA, initiator of radiocarbon activity in Florence.

\section{Nicla Gelli}

National Institute of Nuclear Physics (INFN), Florence, Italy. Physicist/researcher working in nuclear and applied nuclear physics. Expertise in IBA, external microprobe, nuclear physics.

\section{Lorenzo Giuntini}

University of Florence, Department of Physics, Florence, Italy. Associate professor of Physics. Expertise in external microprobe and IBA, initiator of microbeam activity in Florence.

\section{Novella Grassi}

University of Turin, Department of Physics, Turin, Italy. Physicist working in applied nuclear physics. Expertise in IBA.

\section{Vladimír Hnatowicz and Vladimír Havránek}

Neutron Physics Department, CANAM (Centre for Accelerators and Nuclear Analytical Methods), Nuclear Physics Institute of the Czech Academy of Sciences, Řež, Czech Republic. Ion beam applications for material science, nanostructures deposition and characterisation, microbeam applications, archaeometry, environment studies. Experience in simulation, polymer degradation by irradiation and interaction of energetic ions with materials.

\section{Violeta Iancu}

Extreme Light Infrastructure - Nuclear Physics, Horia Hulubei National Institute for R\&D in Physics and Nuclear Engineering, Bucharest-Magurele, Romania. Junior researcher. Expertise in experimental applied physics, nanostructure deposition and characterisation and numerical simulations.

\section{Mihai Iovea}

ACCENT PRO 2000 s.r.l., Research \& Development Company, Romania. Expert in the field of X-ray imaging, industrial X-ray tomography and X-ray diffraction.

\section{Milko Jaksic}

Ruđer Bošković Institute, Division of experimental physics, Laboratory for ion beam interactions and Centre of excellence for materials and sensing devices (CEMS) Zagreb, Croatia. Senior scientist. Expertise in development and applications of ion microbeam techiques for material modification and analysis.

\section{Zsolt Kasztovszky}

Nuclear Analysis and Radiography Department MTA Centre for Energy Research, Budapest, Hungary. Expertise in prompt gamma analysis after neutron activation.

\section{Anabelle Kriznar}

Centro Nacional de Aceleradores CNA, Universidad de Sevilla, Spain. Interests and expertise: Art History and Hispanic language. Currently a researcher at the University of Seville, analysing $15^{\text {th }}$ and $16^{\text {th }} \mathrm{C}$. panel paintings and polychrome sculpture at the Fine Arts Museum of Seville using non-destructive techniques (UV light, IR spectroscopy, X-Ray fluorescence). 


\section{Jan Kučera}

Neutron Physics Department, Nuclear Physics Institute of the Czech Academy of Sciences, CANAM (Centre of Accelerators and Nuclear Analytical Methods), Rež, Czech Republic. Expertise in nuclear analytical methods, namely neutron activation analysis and its applications in material science, archaeometry, environmental geochemical and biomedical studies, and in preparation and certification of reference materials.

\section{Walter Kutschera}

Emeritus Professor of Physics at the University of Vienna, Austria. Research fields: archaeology, art, atmospheric science, atomic and molecular physics, biomedicine, environmental physics, forensic medicine, Egyptology, geochronology, geomorphology, geophysics, glaciology, groundwater dating, nuclear astrophysics, nuclear physics, oceanography, paleoclimatology.

\section{Lucia Liccioli}

University of Florence, Department of Chemistry, Department of Physics, Florence, Italy. Scientist for cultural heritage. PhD student with expertise in radiocarbon.

\section{Franco Lucarelli}

University of Florence, Department of Physics, Florence, Italy. Associate professor of Physics. Expertise in IBA and environmental studies. Initiator of IBA for aerosol studies in Florence.

\section{Anna Macková}

Neutron Physics Department, CANAM (Centre of Accelerators and Nuclear Analytical Methods), Nuclear Physics Institute of the Czech Academy of Sciences, Řež, Czech Republic. Nuclear physicist working with a wide range of ion beams for material science, nanostructure deposition and characterisation, microbeam applications, archaeometry and environment studies. Associate professor at the J.E. Purkynje University, Usti nad Labem, Czech Republic.

\section{Pier Andrea Mandò}

University of Florence, Department of Physics, Florence, Italy. Professor of Physics working in applied nuclear physics. Expertise in radiocarbon and IBA, initiator of IBA in Florence.

\section{Mirko Massi}

National Institute of Nuclear Physics (INFN), Florence, Italy. Physicist in the field of applied nuclear physics; working position: technologist; expertise in microprobes and IBA, initiator of microbeam activity in Florence.

\section{Anna Mazzinghi}

University of Florence, Department of Physics, scientist for cultural heritage, working position: $\mathrm{PhD}$ student, expertise in diagnostics for $\mathrm{CH}$.

\section{Douglas MacGregor}

University of Glasgow, United Kingdom. Professor of Physics. Vice-chair EPS Nuclear Physics Division; chair EPS committee for IBA prize for Applied Nuclear Science and Nuclear Methods in Medicine; expertise in intermediate energy photonuclear physics.

\section{Alessandro Migliori}

International Atomic Energy Agency, Vienna, Austria. Physicist working in applied nuclear physics; expertise in IBA.

\section{Valentin Moise}

Horia Hulubei National Institute for Nuclear Physics and Engineering, Bucharest, Romania. Experience - gammarays disinfection and radio-polymerisation for wooden artefacts consolidation.

\section{Alessandro Re}

University of Turin, Department of Physics, Turin, Italy. Physicist, research fellow, working in applied material science; expertise in science for cultural heritage.

\section{Miguel Angel Respaldiza}

University of Seville, Sevilla, Spain. Full Professor in Atomic, Molecular and Nuclear Physics Department, Director of the National Accelerator Centre from its creation till 2005, Scientific Adviser to the Centre 2005-2009. Co-ordinator of the "Network Science and Heritage" at the University of Seville. Research in $X$-Ray Fluorescence spectrometry: milli-, micro- and confocalXRF; non-destructive analysis of Cultural Heritage objects, IBA applications in archaeometry, biomedicine, environment and material science. Gamma-ray spectrometry.

\section{László Rosta}

Department of Neutron Spectroscopy, Wigner Research Centre For Physics, Budapest, Hungary. A senior expert in neutron scattering methods (SANS, TOF-ND, etc.).

\section{Chiara Ruberto}

University of Florence, Department of Chemistry, Florence, Italy. Scientist for cultural heritage, working position: $\mathrm{PhD}$ student. Expertise in diagnostics for cultural heritage.

\section{Francisco Javier Santos}

Centro Nacional de Aceleradores CNA, Universidad de Sevilla, Consejo Superior de Investigaciones Científicas CSIC, Junta de Andalucía, Spain. Physicist in the AMS Unit and one of the heads of the Radiocarbon unit. Research fields: the use and technical maintenance of two AMS accelerators (SARA and Micadas) at CNA, since 2005.

\section{Simona Scrivano}

Centro Nacional de Aceleradores CNA, Molecular and Nuclear Physics Department, Universita Sevilla, Spain. The development of portables devices for the analysis of Cultural Heritage and the study of archaeological metallic objects. 


\section{Zdravko Siketić}

Ruđer Bošković Institute, Division of experimental physics, Laboratory for ion beam interactions and Centre of excellence for materials and sensing devices (CEMS) Zagreb, Croatia. Research associate with expertise in development and applications of depth profiling and surface analysis techniques based on ion beams.

\section{Gabriel Suliman}

Extreme Light Infrastructure - Nuclear Physics, Horia Hulubei National Institute for R\&D in Physics and Nuclear Engineering, Bucharest-Magurele, Romania. Junior researcher; expertise in experimental nuclear physics and numerical Monte Carlo simulations.

\section{Žiga Šmit}

Faculty of Mathematics and Physics, University of Ljubljana, and Jožef Stefan Institute, Ljubljana, Slovenia. PIXE,PIGE and microbeam analysis of archaeological artefacts, geological and environmental samples, expert in the field of ion beam analysis of various kinds of archaeological artefacts.

\section{Zoltán Szökefalvi-Nagy}

MTA Wigner Research Centre for Physics, Budapest, Hungery. Research professor emeritus, expertise in development and application of PIXE, XRF and other IBA techniques.

\section{Francesco Taccetti}

National Institute of Nuclear Physics (INFN), Florence, Italy. Physicist; applied nuclear physics and electronic; working position: technologist; expertise in radiocarbon and IBA.

\section{Calin Alexandru Ur}

Extreme Light Infrastructure - Nuclear Physics, Horia Hulubei National Institute for R\&D in Physics and Nuclear Engineering, Bucharest-Magurele, Romania. Senior researcher, Head of Research on High-brilliance gammabeam system; expertise in experimental nuclear physics and particle accelerators.

\section{Wasif Zia}

Institut für Technische und Makromolekulare Chemie (ITMC), RWTH Aachen University, Germany. Physicist studying low-field NMR, instruments, methods, and applications. Coauthored the authoritative monograph "Compact NMR" (De Gruyter Berlin, 2014) and is currently working towards miniaturised NMR sensors for everyday use.

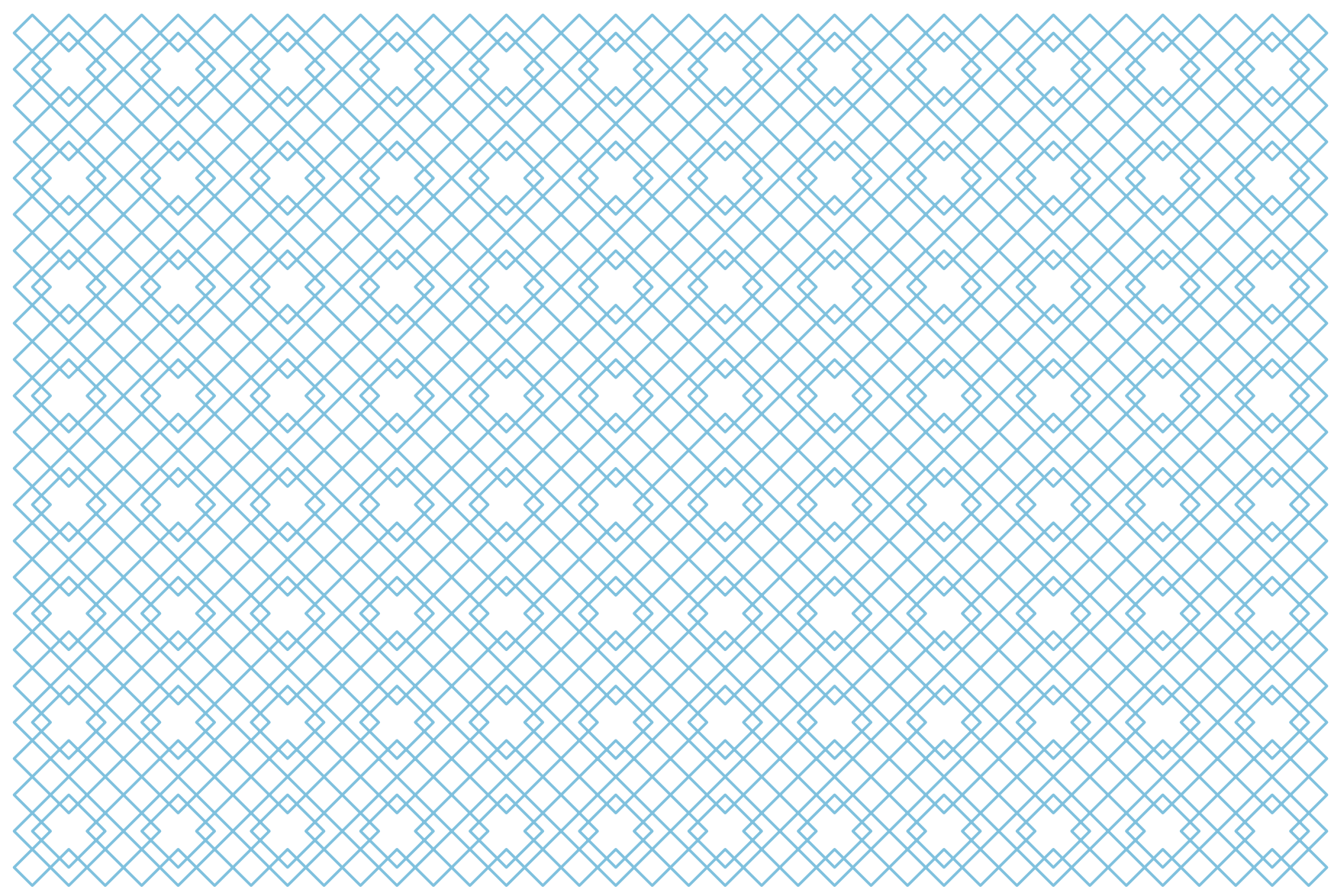

